\title{
Protocolos na atenção à saúde de mulheres em situação de violência sexual sob a ótica de profissionais de saúde
}

\author{
Protocols in healthcare for female victims of sexual violence \\ from the standpoint of healthcare professionals
}

Luiza Jane Eyre de Souza Vieira ${ }^{1}$

Ana Cristina Feijó da Silva ${ }^{2}$

Gracyelle Alves Remigio Moreira ${ }^{1}$

Ludmila Fontenele Cavalcanti ${ }^{3}$

Raimunda Magalhães da Silva ${ }^{1}$

${ }^{1}$ Programa de PósGraduação em Saúde Coletiva, Universidade de Fortaleza. Av. Washington Soares 1321/Bloco S/01, Edson Queiroz. 60811-905 Fortaleza Ceará Brasil. janeeyre@unifor.br ${ }^{2}$ Secretaria de Saúde do Estado do Ceará e Secretaria de Saúde do Município de Fortaleza. Fortaleza CE Brasil.

${ }^{3}$ Programa de PósGraduação em Serviço Social, Universidade Federal do Rio de Janeiro, Rio de Janeiro RJ Brasil.

\begin{abstract}
The use of protocols in health care for women suffering from sexual violence was analyzed from the standpoint of health professionals. It involved a qualitative study conducted in 18 institutions with 140 professionals interviewed in Fortaleza and Rio de Janeiro. After organization of the data, the clusters of meaning pointed to the potential benefits of the use of protocols; limits to be faced in the adoption of protocols; and the need for protocol development and professional training. The results revealed points of convergence and divergence between the two municipalities regarding the use of protocols in health care for women suffering from sexual violence. In Rio de Janeiro, the adoption of protocols in the services by the majority of interviewees and the existence of an established reporting system are acknowledged and involve the work of professionals from different areas. In Fortaleza, the participants of only one institution mentioned the use of protocols and an internal reporting system. In both capitals, professional qualifications to work with women suffering from sexual violence is inadequate due to the limited coverage of the issue during the undergraduate years of the healthcare professions and the lack of training in the health services.
\end{abstract}

Key words Violence against women, Sexual violence, Protocols, Health care
Resumo Analisou-se a utilização de protocolos na atenção à saúde de mulheres em situação de violência sexual sob a ótica de profissionais. Estudo qualitativo com 18 instituições e 140 profissionais entrevistados em Fortaleza e Rio de Janeiro. Diante dos dados organizados os núcleos de sentido apontaram para as potencialidades na utilização de protocolos; limites a serem enfrentados na adoção de protocolos; $e$ necessidade da construção de protocolo e de formação profissional. Os resultados mostraram pontos de convergência e divergência entre os dois municípios em relação à utilização de protocolos na atenção à saúde de mulheres em situação de violência sexual. No Rio de Janeiro é reconhecida a adoção de protocolos nos serviços pela maioria dos entrevistados e a existência de um fluxo estabelecido, envolvendo a atuação das diversas categoriais profissionais. Em Fortaleza, os participantes de apenas uma instituição mencionaram a utilização de protocolos e fluxos internos de atendimento. Nas duas capitais observa-se a fragilidade da qualificação profissional para atuar junto às mulheres em situação de violência sexual decorrente da limitada abordagem durante a graduação das profissões de saúde, agravada pela falta de treinamento nos serviços.

Palavras-chave Violência contra a mulher, Violência sexual, Protocolos, Atenção à Saúde 


\section{Introdução}

Esforços governamentais para o enfrentamento da violência sexual contra a mulher têm culminado em leis, políticas, planos e programas, convocando a sociedade para ressignificar contextos históricos e culturalmente construídos, em que perduram desigualdades de gênero, circunscrevendo a perpetuação dessa modalidade de violência ${ }^{1,2}$.

A Organização Mundial de Saúde (OMS) informa que mais de um terço das mulheres do mundo sofrem agressões físicas ou sexuais, o que representa um desafio global, com proporções epidêmicas. Estes atos ocasionam diversos problemas de saúde, sendo perpetrados, na maioria das vezes, no contexto das relações interpessoais ${ }^{3}$. Reafirma-se que a violência sexual constitui uma das principais formas de violação dos direitos humanos, com graves desdobramentos no cotidiano, estilo de vida e saúde integral, da própria mulher e de seu círculo relacional ${ }^{1,4}$.

No Brasil, a Política Nacional de Enfrentamento à Violência contra as Mulheres baseia-se na intersetorialidade, entre as políticas públicas, na busca de um atendimento integral para sistematizar o enfrentamento à violência sexual garantindo-lhes direitos, em acordo com normas e instrumentos internacionais de direitos humanos e legislação nacional².

A publicação da Norma Técnica "Prevenção e Tratamento dos Agravos Resultantes da Violência Sexual Contra Mulheres e Adolescentes", no âmbito da saúde, vem sendo atualizada para atender os conhecimentos técnicos e as novas demandas de atenção à saúde de mulheres expostas às violências sexuais ${ }^{5,6}$. Este instrumento orienta os serviços de saúde na oferta dessa atenção, preconiza a qualificação dos profissionais e a organização dos serviços; normatiza ações e cuidados integrais em saúde, incluindo a interrupção legal da gravidez ${ }^{6}$.

Na concepção internacional, uma norma técnica é um documento estabelecido por consenso e aprovado por um órgão reconhecido, que fornece diretrizes para atividades visando obter um grau ótimo de ordenação em um dado contexto. Nesse sentido, os protocolos facilitam a organização do processo de trabalho, constituídos de um conjunto de regras, padrões e especificações técnicas que regulam os atos públicos ${ }^{7}$.

No campo da saúde, os protocolos são considerados instrumentos normativos de intervenção técnica e social, que orientam os profissionais na realização de suas funções e têm como base os conhecimentos científicos e práticos do coti- diano, adequando-se à realidade e favorecendo a continuidade administrativa e gerencial. A aplicação dos protocolos no Sistema Único de Saúde (SUS) facilita o gerenciamento das ações e o respeito aos princípios de universalidade, equidade e integralidade ${ }^{8}$.

Destarte os esforços globais com as legislações e as normas para sistematizar a atenção às mulheres em situação de violência sexual nos serviços de saúde, nem sempre os profissionais se apropriam dos conteúdos das políticas e documentos protocolares que norteiam a sua prática e que convergem com os princípios administrativos e gerenciais do sistema de saúde. Os profissionais de saúde são corresponsáveis pela produção e gerenciamento direto e indireto de um cuidado integral e humanizado, mesmo sendo a gestão do setor saúde, nos municípios brasileiros, considerada assimétrica quanto à implementação, monitoramento e avaliação de políticas e instrumentos normativos.

Nesse sentido, a relevância deste artigo centra-se na imprescindibilidade da aproximação das concepções dos profissionais sobre o uso de protocolos, considerando que os mesmos operacionalizam as recomendações políticas e normas protocolares dos serviços de saúde que atendem mulheres em situação de violência sexual.

As diretrizes políticas e as estratégias governamentais preconizam que os serviços de saúde ofertem uma atenção respaldada nos princípios epistêmicos e operacionais do SUS. Uma prática centrada nesta abrangência demanda apropriação das estruturas, processos, produtos e prospecções administrativos e gerenciais que ancoram os múltiplos modelos de gestão do setor saúde no Brasil. Nessa perspectiva, torna-se imprescindível aproximar-se das concepções dos profissionais sobre o uso de protocolos, considerando que os mesmos operacionalizam as recomendações políticas e as normas protocolares dos serviços de saúde que atendem mulheres em situação de violência sexual.

Diante desses elementos, procurou-se analisar a utilização de protocolos na atenção à saúde de mulheres em situação de violência sexual sob a ótica de profissionais em duas capitais brasileiras.

\section{Material e método}

Este estudo qualitativo integra uma pesquisa multicêntrica que analisou a atenção à saúde de mulheres em situação de violência sexual em duas capitais do Brasil - Fortaleza e Rio de Ja- 
neiro. A pesquisa foi empreendida em serviços de saúde, de atenção secundária e terciária, da rede municipal, indicados, na época da coleta de dados, como referências para a atenção à saúde às violências sexuais pelas Secretarias Municipais de Saúde (SMS) de Fortaleza e do Rio de Janeiro; envolvendo 18 instituições (nove em cada capital). Vale mencionar que a escolha da instância municipal justifica-se tanto pelo maior número de serviços voltados para a atenção a essa demanda, quanto pelo protagonismo na articulação da rede de serviços locais.

A seleção desses dois municípios pautou-se no fato de representarem contextos culturais diferenciados no que se refere aos indicadores sociais e de violência. De acordo com a Pesquisa de Informações Básicas Municipais (MUNIC) ${ }^{9}$, os serviços especializados que integram a rede de atenção às mulheres em situação de violência sexual concentram-se na Região Sudeste, enquanto a Região Nordeste possui menor disponibilidade de equipamentos. Em relação às violências de gênero perpetradas contra mulheres, a taxa de homicídio é de 5,6 por 100.000 mulheres em Fortaleza (16a posição no País) e de 3,9 no Rio de Janeiro (22a posição no País $)^{10}$. Os registros estaduais da segurança pública, do ano de 2012, revelam taxa de 17,2 (1.483 casos) referente aos crimes contra a liberdade sexual no Ceará e de 36,5 (5.923 casos) no Rio de Janeiro ${ }^{11}$. Quanto às notificações das violências sexuais contra as mulheres realizadas pelo setor saúde, Fortaleza registrou 148 notificações e o município do Rio de Janeiro empreendeu 2.014, no período de 2009 e $2013^{12}$.

Participaram do estudo profissionais de saúde de nível superior, integrantes da equipe multiprofissional, envolvidos na atenção às mulheres em situação de violência sexual nos referidos serviços. Considera-se que esses profissionais são os agentes centrais na tradução de políticas públicas em práticas nos serviços de saúde, justificando o propósito de aproximar-se de uma realidade descrita sob a perspectiva desses participantes.

A constituição do conjunto de participantes foi baseada na experiência em relação à atenção à violência sexual, na diversidade e no acesso. Ao todo foram entrevistados 140 profissionais (68 em Fortaleza e 72 no Rio de Janeiro), com participação média de oito trabalhadores da saúde por serviço. A composição da categoria profissional abrangeu: enfermeiros (39), médicos (38), assistentes sociais (37), psicólogos (25) e pedagoga (01).
Para a produção de dados utilizou-se a entrevista semiestruturada abordando questões relativas à identificação e formação profissional, inserção institucional, qualificação para a atenção à saúde de mulheres em situação de violência sexual, utilização de protocolos e fluxos. A captação dos profissionais para as entrevistas se deu por intermédio de indicação interpessoal dos trabalhadores das instituições. Estes recomendavam colegas da equipe multiprofissional com maior experiência na atenção às mulheres em situação de violência sexual, formando uma rede de relações entre os investigadores e os interlocutores.

As entrevistas foram realizadas no período de agosto a dezembro de 2013, com consentimento assinado pelos informantes, de forma individual, em ambiente reservado, em dia e horário favorável para cada participante. As gravações tiveram média de 30 minutos por entrevista, sendo posteriormente transcritas na íntegra e codificadas com as siglas PF (Profissional Fortaleza) e PR (Profissional Rio de Janeiro) e numeradas de 1 a 140 , como forma de preservar o anonimato dos participantes.

Após a transcrição, realizou-se leitura de cada entrevista e procedeu-se a organização do material empírico, decompondo-o em partes correspondentes aos aspectos abordados no roteiro e as respectivas falas dos participantes. Analisouse este conjunto de dados segundo a técnica de análise de conteúdo na modalidade temática ${ }^{13}$, guiando-se pela trajetória analítica proposta por Gomes $^{14}$.

De posse da organização do material, realizou-se leitura detalhada identificando-se os sentidos atribuídos pelos participantes em cada parte do esquema de classificação. Posteriormente, foram reagrupados em núcleos centrais, buscando-se temas mais abrangentes e melhor delimitados: potencialidades das práticas profissionais na utilização de protocolos; limites a serem enfrentados na adoção de protocolos; e necessidade da construção de protocolo e de formação profissional. Por fim, buscou-se estabelecer uma compreensão do material empírico articulando-o às diretrizes preconizadas pelas políticas públicas voltadas para a atenção às mulheres em situação de violência sexual e a apreensão das singularidades sobre o uso de protocolos.

A pesquisa foi aprovada pelo Comitê de Ética em Pesquisa da Secretaria Municipal de Saúde e Defesa Civil do Rio de Janeiro. 


\section{Resultados e discussão}

\section{Caracterização dos participantes}

A idade dos profissionais variou de 23 a 66 anos. A maior parte deles, em ambos os municípios, era do sexo feminino, se declarou unido (casamento formal ou união estável) e de religião católica. O tempo de formação variou de um a mais de 25 anos.

Em Fortaleza, predominaram profissionais com 11 a 20 anos de formado e no Rio de Janeiro sobressaíram-se os participantes com mais de 25 anos de profissão. Nos dois municípios, o interesse na qualificação voltada para prática profissional pôde ser evidenciado tendo em vista que quase todos os entrevistados possuíam pós-graduação.

\section{Potencialidades das práticas profissionais na utilização de protocolos}

No Rio de Janeiro, os profissionais, em grande parte das unidades de saúde, reconheceram a adoção de protocolos do Ministério da Saúde especialmente de medicamentos utilizados nas profilaxias das Doenças Sexualmente Transmissíveis (DST) e de anticoncepção de emergência. Por outro lado, em Fortaleza, apenas um pequeno grupo de entrevistados, quase todos vinculados a uma mesma instituição, mencionaram a utilização de protocolos.

Os profissionais das duas capitais descreveram as condutas utilizadas a partir do momento em que a mulher chega aos serviços. Os protocolos que norteiam esses procedimentos são, muitas vezes, adaptações da Norma Técnica do Ministério da Saúde à realidade local. Nos conteúdos manifestos evidencia-se a existência de protocolos distintos para atender as especificidades de cada mulher. Os participantes ressaltaram a importância do atendimento emergencial, a obrigatoriedade do registro e a conduta diante da gestação decorrente da violência sexual realizados por equipe multiprofissional.

Essas apreensões confirmam-se nas falas: $O$ fluxo é assim: entra na unidade, é feita toda a profilaxia para DST. Passa pela enfermagem, sendo gestação, passa pelo médico, serviço social e saúde mental. (PR 73). Existem dois fluxos. Quando a vítima chega até 72 horas, preenche a ficha epidemiológica, encaminha para a realização das profilaxias e anticoncepção de emergência. Quando ela já vem grávida e faz o pedido do aborto legal, o serviço social e a psicologia trabalham essa escolha [ $\mathrm{da}$ mulher], tiram as dúvidas dela. A gente cuida dela antes, durante e depois do aborto. (PF 18).

A construção de um protocolo municipal ou institucional reflete os esforços dos gestores locais na implementação das políticas, no planejamento e monitoramento das ações, favorecendo as atividades de gerenciamento, as articulações de saberes e práticas das diferentes categorias profissionais no sentido de efetivar as ações intersetoriais.

A adoção de protocolos permite que os profissionais tenham subsídios para realizar um atendimento mais preciso e eficiente diante dos casos de violência ${ }^{15,16}$. Estes também são essenciais para discernir o julgamento clínico da terapêutica medicamentosa e do fluxo interno hospitalar, ou de outros setores, após a mulher ter sido atendida ${ }^{17}$.

Nessa ótica, os protocolos devem ser baseados em evidências clínicas e epidemiológicas, revistos periodicamente e amplamente divulgados aos trabalhadores da saúde. Esses instrumentos precisam indicar os serviços de referência, o fluxo de atendimento, os medicamentos necessários, as orientações e as informações que devem ser fornecidas, dentre outros aspectos. Nessa perspectiva, a adoção de protocolos imprime qualidade às ações de cuidado e de gestão, viabilizando uma atenção que responda apropriadamente às mulheres em situação de violência sexual.

Em contrapartida, apesar de ser um instrumento de grande valia para a organização dos serviços de saúde, os protocolos não respondem a todas as questões e nem sempre representam uma alternativa para o direcionamento de situações imprevistas ${ }^{18}$. Silva et al. ${ }^{19}$ advogam a necessidade de se ponderar seu uso, uma vez que a complexidade do objeto violência requer soluções plurais, por isso, algumas vezes, projetos assistenciais técnicos baseados em protocolos fixos e únicos não promovem resultados eficientes. Em detrimento disso, ressalta-se que em virtude das peculiaridades de cada caso, a atenção à saúde dessas mulheres não deve se restringir ao que preconizam os protocolos, ao contrário, deve transpor a burocratização e reafirmar uma atenção integral e resolutiva.

Outros pontos identificados nas falas dos participantes dizem respeito à valorização do trabalho em equipe, sintonizados com a compreensão de que a produção do cuidado em saúde requer, cada vez mais, ações integradas entre os profissionais, ao se considerar que as demandas que chegam neste setor implicam em desdobramentos mais complexos. Ainda é importante reconhecer que a perspectiva de uma atenção 
integral está posta nos conteúdos manifestos dos participantes.

Depreende-se que esta atenção precisa "acolher a violência” em sua complexidade, pensar possibilidades de não violência, prevenir novas ocorrências e tratar as consequências. Nesta premissa, há necessidade de considerar a mulher usuária como centro da tomada das decisões. Sendo a violência um tema complexo, demanda a multiprofissionalidade e a intersetorialidade da atenção ${ }^{20,21}$.

A oferta dessa atenção integral também implica que profissionais e instituições compreendam as delimitações do objeto de intervenção do outro com quem pretendem compor equipes e, dessa forma, construam um objeto integral compartilhado $^{21}$.

\section{Limites a serem enfrentados na adoção de protocolos}

A inexistência e o desconhecimento dos documentos protocolares, assim como a não divulgação daqueles instituídos foram apontados pelos profissionais dos dois municípios, com mais expressão em Fortaleza, como dificuldades da atenção à saúde de mulheres em situação de violência sexual.

Os depoimentos dos participantes transparecem a insegurança e a inabilidade de suas práticas frente aos casos de violência sexual em virtude da limitada ou ausente qualificação acerca da questão. Se existe esse fluxo escrito, eu desconheço [...] existe uma necessidade [...] As pessoas não foram treinadas. Existe uma dificuldade do atendimento por isso. Os serviços precisam atender, mas não sabem muito bem sua função ainda [...]. (PR 113). Esse fluxo deveria estar disponível e visivel, para quando a paciente chegar, o plantonista saber os passos que devem ocorrer (PF 13).

Esses aspectos que se reportam ao desconhecimento podem indicar ínfimo investimento da gestão de alguns hospitais na educação permanente de seus recursos humanos e o não desenvolvimento de instrumentos normativos que orientem a prática profissional diante dos casos de violência sexual. Supõe-se que a inexistência de protocolo ou sua insuficiente aplicação em Fortaleza seja pela recente trajetória de implementação dessa atenção no conjunto de unidades da rede pública de saúde e pelo incipiente preparo dos profissionais na abordagem do fenômeno.

A não utilização de protocolos pode limitar a atuação profissional nesse tipo de atenção, uma vez que favorece o desconhecimento do fluxo de atendimento, acarretando entraves na realização dos procedimentos indicados, da notificação e dos encaminhamentos necessários para cada situação.

Importante frisar que no Brasil, protocolos e normatizações que orientam as ações de saúde são recentes e provavelmente não estão completamente disseminados pelos serviços existentes ${ }^{21}$. É recorrente na literatura o discurso dos profissionais quanto à inabilidade de conhecimento $\mathrm{e}$ de atitude para lidar com as pessoas em situação de violências ${ }^{15,17,19}$.

Em contraponto, a inclusão das violências nas agendas do setor saúde deu-se na década de 90 e, desde então, os governos têm investido na produção de material educativo, cursos de formação à distância, manuais, normas técnicas, dentre outros dispositivos, no sentido de minimizar as lacunas de conhecimentos decorrentes de uma formação que ainda não incluía nas matrizes curriculares conteúdos que discorram e problematizem situações limite da realidade sociosanitária dos municípios brasileiros.

Esse descompasso que perdura nas práticas assistenciais nos municípios estudados poderia ser minimizado por uma gestão articulada com as políticas e ancorada pela legislação, alicerçada nos protocolos, complementada com monitoramento e avaliação sistemáticos das ações instituídas, para preservar a saúde das mulheres em situação de violência sexual. Vale pontuar que o "instituído" no contexto de uma gestão compartilhada deve ser flexível e receptivo às possíveis mudanças organizacionais em virtude da dinâmica do fenômeno.

O emprego responsável e regular de protocolos deve ser consequência de um processo de planejamento, gerando a necessidade de que os gestores estejam atentos e deflagrem um acompanhamento constante, avaliando a efetividade desses instrumentos e as relações produzidas por este processo. Na sua utilização, considera-se o pacto entre os atores do processo assistencial, sendo oportuno que haja um acordo de funcionamento entre todas as esferas participantes ${ }^{22}$.

Vale referir que a gestão institucional de cada unidade hospitalar é responsável em prover sua força de trabalho em saúde de conhecimentos científicos e instrumentais, de modo permanente, haja vista as modalidades de contratualização, renovação, absenteísmo e a precarização dos vínculos profissionais que tem caracterizado o setor saúde dos municípios.

Em Fortaleza, além de muitos profissionais desconsiderarem o uso dos protocolos, outros 
verbalizaram a fragmentação e a inadequação da organização da rede de serviços de saúde. Fato observado em uma unidade de saúde, a qual disponibiliza os medicamentos para as profilaxias e a anticoncepção de emergência e demais procedimentos, a não incorporação do atendimento às mulheres em situação de violência sexual como demandante de medidas emergenciais, apesar de ter sido gestada com uma filosofia de atenção integral à saúde da mulher e seus direitos reprodutivos. Aqui tem o protocolo de violência sexual do hospital. Tem a anticoncepção de emergência, os exames de sangue, cirurgia. Mas, a gente não atende emergência, esse é o nosso dilema. Temos o material, mas como não atendemos emergência, o que é que na prática a gente faz? O atendimento a nivel crônico, não as medidas emergenciais da violência sexual propriamente. (PF 50).

Esta forma de organização da rede de serviços não contribui para o aprimoramento dos atendimentos, podendo anular a intenção da aplicação do protocolo efetivo. Chama à atenção o paradoxo referente à estruturação do Hospital da Mulher, idealizado sob as diretrizes da atenção integral à saúde da mulher, dispor de insumos e equipamentos e não ofertar o atendimento emergencial às mulheres em situação de violência sexual. Esse fato pode estar relacionado com a inauguração relativamente recente, no final da gestão governamental anterior, agravado pela descontinuidade entre as gestões ${ }^{23}$.

Sobre a realização do aborto legal identificase nos dois municípios a incipiente oferta desse serviço, pois apenas uma instituição em cada o realiza. Em Fortaleza, essa lacuna torna-se mais agravante porque o hospital que o executa restringe a realização a um único dia da semana e a dinâmica de trabalho de apenas uma equipe profissional. O aborto é realizado aqui mesmo e ela marca com a gente na quarta à tarde. Quarta-feira é o único dia que a equipe está completa e que o médico faz o aborto legal. (PF 18).

A organização desse processo de trabalho, concentrando a interrupção legal da gravidez ao encargo de "uma única equipe e um único profissional", fere as prerrogativas legais, os direitos assegurados e as orientações do Ministério da Saúde. No Brasil, o cumprimento do aborto previsto em lei ainda esbarra em dificuldades administrativas e de ordem pessoal dos profissionais de saúde ${ }^{24,25}$. Apesar de a atenção às mulheres em situação de violência sexual ter trazido, com mais ênfase, o tema do aborto para o cotidiano dos serviços de saúde, essa questão ainda se configura como tabu para muitos profissionais.
Vale esclarecer que a objeção de consciência para a não realização da interrupção da gravidez prevista em lei deve ser respeitada, no entanto, as instituições necessitam oferecer condições para tal prática disponibilizando outros profissionais. A negação dessa prática pode levar a mulher a buscar um aborto em condições inseguras, o que se constitui em um problema ainda maior com sérias repercussões à sua saúde ${ }^{26}$.

\section{Necessidade da construção de protocolo e de formação profissional}

Na descrição dos entrevistados acerca da utilização de protocolos na atenção à saúde de mulheres em situação de violência sexual, nas duas capitais, percebem-se respostas evasivas, desconexas e sem um julgamento crítico do contexto do atendimento. Mostraram-se recorrentes as respostas monossilábicas que aparentavam total desconhecimento, que o profissional não pertencia ao cenário de cuidado e que aquele tema não lhe dizia respeito. Em outros momentos, as verbalizações se assemelhavam a novas indagações, pressupondo uma produção acrítica do cuidado: tem, não tem, não sei, desconheço, é para ter?; se tem não conheço e deveria ter.

No cenário de Fortaleza, os participantes que referem a existência de protocolos, reportam-se sobre o seu modo informal, pontual e imbuído de atitudes pessoais do profissional. A maioria dos entrevistados restringiu o protocolo da atenção às violências sexuais ao ato notificatório, reduzindo-o ao mero preenchimento de dados para compor uma ficha e alimentar o Sistema de Informação de Agravos de Notificação (SINAN). Não sei, mas eu anoto no meu instrumento particular. (PF 28). Tem uma notificação. Tem uma ficha que a gente levanta todos os dados. Preenche essa notificação e encaminha para o setor da epidemiologia. (PF 9).

Não se desconsidera a relevância desta etapa, que qualifica os registros e proporciona a visibilidade e a magnitude do fenômeno, entretanto, o seguimento de um protocolo não se limita a este procedimento. Pelo contrário, tem uma interlocução direta com uma atenção centrada no sujeito, apreendendo especificidades e singularidades do atendimento.

O protocolo de Manchester também foi mencionado por alguns profissionais vinculados aos serviços de referência na área de traumato-ortopedia no município de Fortaleza. Este protocolo é aplicado para situações gerais, com ênfase na avaliação de risco, e classifica os pacientes de 
acordo com a gravidade da situação e o tempo de espera. A gente segue o protocolo de Manchester. Ele é geral, para vários pacientes. (PF 37).

No entanto, a avaliação de risco preconizada pela Norma Técnica ${ }^{6}$ diz respeito à análise das múltiplas condições que tornam a mulher mais exposta às ocorrências da violência sexual. Nessa perspectiva, o protocolo de Manchester não se aplica à atenção a essas mulheres que buscam os serviços de saúde.

Há de se considerar a característica dos hospitais denominados "Frotinhas" (referência na área de traumato-ortopedia), que foram instituídos para o atendimento emergencial ao politraumatizado e cujo investimento na formação do profissional incorreu sobre os níveis de complexidade dos cuidados para manter os parâmetros vitais e nos suportes de vida. Por outro lado, não se justifica que a gestão institucional mantenhase à margem dos avanços legais que subsumem a atenção à saúde. A Lei no $12.845 / 2013^{27}$, que prevê a obrigatoriedade do atendimento integral a pessoas em situação de violência sexual em todos os hospitais integrantes do SUS, requer reestruturação do processo de trabalho para essa demanda.

Ainda no município de Fortaleza também foi notório os discursos que associaram o atendimento prioritário das ocorrências de violências ao escopo da formação acadêmica e da prática profissional do assistente social imputando a essa categoria a responsabilidade pelo acolhimento e encaminhamentos preconizados pela Norma Técnica ${ }^{6}$. Eu acredito que o serviço social, na questão da enfermagem não. (PF 12). Esse fluxo quem tem é o serviço social. Eles já têm todos os telefonemas, sabem quem acionar, como orientar e encaminhar a mulher. (PF 8).

Esse posicionamento reflete, por um lado, um distanciamento dos demais profissionais da dimensão da saúde como um direito social, e, por outro, um reconhecimento das fragilidades no campo da formação profissional.

Dessa maneira, a prática profissional se insere em uma linha descontínua em que a usuária é atendida por categorias profissionais desarticuladas. A fragmentação da organização do trabalho instaura um modelo verticalizado em que cada categoria e especialidade institui seu modo de atuar. A falta de preparação na formação acadêmica para esse tipo de atendimento se traduz em profissionais que ainda não conseguem trabalhar em uma perspectiva inclusiva, emancipatória e intersetorial ${ }^{17}$.

Estudiosos do tema reforçam que a integralidade das ações, nesse âmbito, só se efetiva quan- do profissionais e serviços tiverem consciência do caráter parcelar de seu objeto e de suas ações e estabelecerem nexos produtivos entre si, compondo um trabalho coletivo na produção assistencial $^{21}$.

\section{Considerações finais}

Apesar dos pontos de convergência, os resultados apontam para uma incorporação diferenciada nos dois municípios em relação à utilização de protocolos na atenção à saúde de mulheres em situação de violência sexual.

No Rio de Janeiro, é reconhecida a adoção de protocolos nos serviços investigados pela maioria dos participantes. Também se evidenciou a existência de um fluxo estabelecido e a atuação das diversas categoriais profissionais. Em Fortaleza, somente os participantes de uma instituição mencionaram a utilização de protocolos e fluxos internos de atendimento às mulheres em situação de violência sexual. Em contrapartida, é expressivo o desconhecimento, a não compreensão e a não adoção de protocolos nesse município, o que aponta para a tímida aproximação da gestão local às políticas públicas que norteiam essa atenção.

Nas duas capitais, observa-se fragilidade da qualificação profissional para esse tipo de atuação, decorrente da limitada abordagem sobre esse tema durante a graduação das profissões de saúde, agravada pela falta de treinamento no contexto dos serviços; o que contribui para a invisibilidade da demanda e para uma assistência nem sempre adequada.

Há necessidade de investimento e ampliação das atualizações e acompanhamentos continuados dos profissionais treinados, incluindo gestores e profissionais de outras áreas, que além do enfoque no preparo técnico, deve envolver as proteções legais e a avaliação de risco. É importante ainda que a gestão tenha a disponibilidade para ouvir o cliente interno, aqui representado pelas categorias profissionais, orientar as diretrizes políticas e as ações normativas, debatendo-se, amplamente, a função social dos profissionais e dos serviços. Possivelmente, instituir a prática de um planejamento ascendente contribua para reduzir os conhecimentos ancorados no senso comum ainda presente nas falas dos entrevistados sobre a utilização de protocolos na compreensão do fenômeno da violência sexual contra a mulher.

A despeito das singularidades presentes nos diferentes serviços de saúde, torna-se necessário qualificar o cuidado integral às mulheres em 
situação de violência sexual a partir da construção de protocolos compartilhados dirigidos à garantia de direitos no campo da saúde sexual e reprodutiva.

Ressalta-se, contudo, que efetivar a integralidade do sistema de saúde extrapola o desejo individual. A institucionalização de protocolos, como instrumento dinâmico de planejamento, envolve um engajamento de diferentes instâncias, como a gestão municipal, as gestões dos serviços, os agentes formadores e o movimento organizado de mulheres.

\section{Colaboradores}

LJES Vieira, ACF Silva e GAR Moreira participaram de todas as etapas da elaboração do artigo. LF Cavalcanti coordenou o desenvolvimento da pesquisa e realizou a revisão crítica do artigo. RM Silva contribuiu com a revisão crítica do artigo.

\section{Agradecimentos}

Ao Conselho Nacional de Desenvolvimento Científico e Tecnológico (CNPq), Edital MCTI/ CNPq/SPM-PR/MDA - Relações de Gênero, Mulheres e Feminismos; e à Fundação Cearense de Apoio ao Desenvolvimento Científico e Tecnológico (FUNCAP), Bolsa Doutorado. 


\section{Referências}

1. Contreras JM, Bott S, Guedes A, Dartnall E. Violência sexual na América Latina e no Caribe: uma análise de dados secundários. Iniciativa de Pesquisa sobre violência sexual, 2010 mar [acessado 2015 jun 15]; [cerca de 100p.]. Disponível em: http://www.svri.org/ViolenciaSexualnaALenoCaribe.pdf

2. Brasil. Presidência da República (PR). Política Nacional de Enfrentamento à Violência contra as Mulheres. Brasília: PR; 2011.

3. World Health Organization (WHO). Global and regional estimates of violence against women: prevalence and health effects of intimate partner violence and nonpartner sexual violence. Geneva: WHO; 2013.

4. Facuri CO, Fernandes AMS, Oliveira KD, Andrade TS, Azevedo RCS. Violência sexual: estudo descritivo sobre as vítimas e o atendimento em um serviço universitário de referência no Estado de São Paulo, Brasil. Cad Saude Publica 2013; 29(5):889-898.

5. Brasil. Ministério da Saúde (MS). Prevenção e tratamento dos agravos resultantes da violência sexual contra mulheres e adolescentes: normas técnicas. Brasília: MS 1999.

6. Brasil. Ministério da Saúde (MS). Prevenção e tratamento dos agravos resultantes da violência sexual contra mulheres e adolescentes: norma técnica. $3^{\text {a }}$ ed. Brasília: MS; 2012.

7. Krause W, Bastos A. As Normas Técnicas em benefício das melhores práticas. Mundo Project Management [periódico na Internet]. 2009 Fev-Mar [acessado 2015 jun 07];5(25): [cerca de 4 p.]. Disponível em: http://www. mundopm.com.br/pres_reved25.shtml

8. Hebling SRF, Pereira AC, Hebling E, Meneghim MC. Considerações para elaboração de protocolo de assistência ortodôntica em saúde coletiva. Cien Saude Colet 2007; 12(4):1067-1078.

9. Instituto Brasileiro de Geografia e Estatística (IBGE). Perfil dos municípios brasileiros 2009. Rio de Janeiro: IBGE; 2010

10. Waiselfisz JJ, organizador. Mapa da Violência 2012 Atualização: homicídio de mulheres no Brasil. São Paulo: Flacso; 2012.

11. Fórum Brasileiro de Segurança Pública. Anuário Brasileiro de Segurança Pública. São Paulo: Fórum Brasileiro de Segurança Pública [Internet]. 2013 mar-abr [acessado 2015 abr 02]; [cerca de 136p.]. Disponível em: http://www.forumseguranca.org.br/storage/download//anuario_2013-corrigido.pdf.

12. Brasil. Ministério da Saúde (MS). Sistema de informação de agravos de notificação - SINAN. [acessado 2015 maio 05]. Disponível em: http://dtr2004.saude.gov.br/ sinanweb/.

13. Minayo MCS, organizador. O desafio do conhecimento: pesquisa qualitativa em saúde. $13^{\text {a }}$ ed. São Paulo: Hucitec; 2013.

14. Gomes R. Análise e interpretação de dados de pesquisa qualitativa. In: Minayo MCS, Deslandes SF, Gomes R, organizadores. Pesquisa social: teoria, método e criatividade. 33a ed. Petrópolis: Vozes; 2013. p. 79-108.
15. Hasse M, Vieira EM. Como os profissionais de saúde atendem mulheres em situação de violência? Uma análise triangulada de dados. Saúde em Debate 2014; 38(102):482-493.

16. García-Moreno C, Hegarty K, D’Oliveira AFL, Koziol-McLain J, Colombini M, Feder G. The health-systems response to violence against women. Lancet 2015; 385(9977):1567-1579.

17. Pedrosa CM, Spink MJP. A violência contra mulher no cotidiano dos serviços de saúde: desafios para a formação médica. Saude Soc 2011; 20(1):124-135.

18. Werneck MAF, Faria HP, Campos KFC. Protocolos de cuidado à saúde e de organização do serviço. Belo Horizonte: Editora Coopmed; 2009.

19. Silva EB, Padoin SMM, Vianna LAC. Mulher em situação de violência: limites da assistência. Cien Saude Colet 2015; 20(1):249-258.

20. D’Oliveira AFPL, Schraiber LB, Hanada H, Durand J. Atenção integral à saúde de mulheres em situação de violência de gênero - uma alternativa para a atenção primária em saúde. Cien Saude Colet 2009; 14(4):10371050.

21. Hanada H, D’Oliveira AFPL, Schraiber LB. Os psicólogos na rede de assistência a mulheres em situação de violência situação de violência. Estudos Feministas 2010; 18(1):33-60

22. Franco CM, Franco TB. Linhas do Cuidado Integral: Uma proposta de organização da rede de saúde. In: $S e$ cretaria de Estado de Saúde do RS. [página na Internet]; [acessado 2014 fev 13]. Disponível em: http://www. saude.rs.gov.br/dados/1306960390341linha-cuidado -integralconceito-como-fazer.pdf.

23. Minayo MCS. Conceito de avaliação por triangulação de métodos. In: Minayo MCS, Assis SG, Souza ER. Avaliação por triangulação de métodos - Abordagens de programas sociais. $2^{\circ}$ ed. Rio de Janeiro: Fiocruz; 2005.p. 19-51.

24. Diniz D. Violência sexual e saúde. Posfácio. Cad Saude Publica 2007; 23(2):477-478.

25. Villela WV, Lago T. Conquistas e desafios no atendimento das mulheres que sofreram violência sexual. Cad Saude Publica 2007; 23(2):471-475.

26. Bedone AJ, Faúndes A. Atendimento integral ás mulheres vítimas de violência sexual: Centro de Assistência Integral à Saúde da Mulher, Universidade Estadual de Campinas. Cad Saude Publica 2007; 23(2):465-469.

27. Brasil. Lei $n^{\circ} 12.845$, de $1^{\circ}$ de agosto de 2013. Dispõe sobre o atendimento obrigatório e integral de pessoas em situação de violência sexual. Diário Oficial da União 2013; 1 ago.

Artigo apresentado em 13/08/2015

Aprovado em 26/10/2015

Versão final apresentada em 28/10/2015 